\title{
Multicenter experience from the UK and Ireland of use of lumen- apposing metal stent for transluminal drainage of pancreatic fluid collections $\square$
}

\section{(๑) $\odot \ominus$}

\section{Authors}

Suresh Vasan Venkatachalapathy', Noor Bekkali², Stephen Pereira ${ }^{3,4}$, Gavin Johnson ${ }^{4}$, Kofi Oppong², Manu Nayar², John Leeds ${ }^{2}$, Bharat Paranandi ${ }^{5}$, Ian Penman ${ }^{6}$, Nicholas Carroll7, Edmund Godfrey ${ }^{7}$, Martin James ${ }^{1}$, Guruprasad Aithal ${ }^{1}$, Colin McKay ${ }^{8}$, John Devlin ${ }^{9}$, Terry Wong ${ }^{10}$, Alistair Makin ${ }^{11}$, Barbara Ryan ${ }^{12}$, Matthew Huggett ${ }^{5}$

Institutions

1 Nottingham University Hospitals NHS Trust And University Of Nottingham - Nottingham Biomedical Research Centre and NIHR Nottingham Biomedical Research Centre, Nottingham, United Kingdom of Great Britain and Northern Ireland

2 Freeman Hospital - HPB Unit, Newcastle upon Tyne, Newcastle upon Tyne, United Kingdom of Great Britain and Northern Ireland

3 University College London - UCL Institute of Hepatology, London, London, United Kingdom of Great Britain and Northern Ireland

4 University College Hospitals NHS Foundation Trust Department of Gastroenterology, London, London, United Kingdom of Great Britain and Northern Ireland

5 Leeds Teaching Hospitals NHS Trust Gastroenterology, Leeds, Leeds, United Kingdom of Great Britain and Northern Ireland

6 Royal Infirmary of Edinburgh- Gastroenterology, Edinburgh, Edinburgh, United Kingdom of Great Britain and Northern Ireland

7 Cambridge University Hospitals NHS Foundation Trust - Radiology, Cambridge, Cambridgeshire, United Kingdom of Great Britain and Northern Ireland

8 Glasgow Royal Infirmary - Pancreatic Surgery, Glasgow, Glasgow, United Kingdom of Great Britain and Northern Ireland

9 King's College Hospital NHS Foundation Trust Hepatology, London, London, United Kingdom of Great Britain and Northern Ireland

10 Guys and St Thomas' NHS Foundation Trust Gastroenterology, London, United Kingdom of Great Britain and Northern Ireland

11 Central Manchester University Hospitals NHS Foundation Trust - Gastroenterology, Manchester, Greater Manchester, United Kingdom of Great Britain and Northern Ireland

12 Tallaght Hospital/Trinity College Dublin Gastroenterology, Dublin, Ireland submitted 23.8.2017

accepted after revision 22.11.2017

Bibliography

DOI https://doi.org/10.1055/s-0043-125362 |

Endoscopy International Open 2018; 06: E259-E265

(c) Georg Thieme Verlag KG Stuttgart · New York

ISSN 2364-3722

Corresponding author

Suresh Vasan Venkatachalapathy, Nottingham Biomedical Research Centre and NIHR Nottingham Biomedical Research Centre - HPB medicine, Queen's medical centre Derby road Nottingham NG7 2UH, United Kingdom of Great Britain and Northern Ireland

Fax: +011-59-70-9012

suresh.venkatachalapathy@nuh.nhs.uk

\section{ABSTRACT}

Background and study aims Pancreatic fluid collection (PFC) is a common complication of pancreatitis for which endoscopic ultrasound-guided drainage is first-line treatment. A new single-device, lumen-apposing, covered selfexpanding metal stent (LAMS) has been licensed for PFC drainage. We therefore present our multicenter experience with the LAMS for PFC drainage in a multicenter prospective case series to assess success and complication rates.

Patients and methods All adult patients from 11 tertiary centers who had LAMS placement for PFC from July 2015 to July 2016 were included. Data including indications, technical success, clinical success, collection resolution, stent removal, early and late adverse events (AEs), mortality and recurrence at 6 months were collected.

Results 116 patients, median age 52.5 years (range 1680 ) and $67 \%$ male, were treated with a single LAMS in each case. The indication was walled off necrosis (WON) in 70 and pseudocyst in 46 . Median size of the PFC was $11 \mathrm{~cm}$ $(5-21 \mathrm{~cm})$ and the estimated median necrotic volume in 
WON was $30 \%(5 \%-90 \%)$. Stent insertion was technically successful in 115 (99.1\%) and clinically successful in 109 (94\%). Early serious AEs (SAEs): $n=7$ sepsis, $n=1$ stent blockage with food, $n=1$ stent migration requiring laparotomy, $n=1$ stent dislodgement and $n=1$ bleeding requiring
emboliZation. Late AEs: $\mathrm{n}=1$ buried stent and $\mathrm{n}=1$ esophageal fistula. Non-procedure-related deaths: $n=3$ (2.5\%).

Conclusion This multicenter case series demonstrates that use of the new LAMS is feasible, effective and relatively safe in draining PFC with a technical success rate of $99 \%$ and cumulative SAE rate of $11.2 \%$.

\section{Introduction}

The incidence of pancreatic fluid collections (PFC) is $5 \%$ to $16 \%$ and $20 \%$ to $40 \%$ in acute and chronic pancreatitis, respectively [1]. Classification of PFC includes acute peri-pancreatic fluid collection (APFC), acute necrotic collection (ANC), pseudocyst and walled-off pancreatic necrosis (WON). APFC and ANC develop within four weeks of the onset of pancreatitis, usually resolve on their own, and may not need intervention unless secondary infection occurs. Pseudocysts and WON usually develop after 4 weeks and may require intervention if they are symptomatic or become infected.

Historically, PFCs were drained either surgically or percutaneously under radiological guidance. EUS-guided drainage was first reported in 1992 and since then several studies have reported success and complication rates of $80-100 \%$ and $10-$ $20 \%$ respectively [ $2-5$ ] A prospective randomized controlled trial comparing surgical vs. EUS-guided drainage reported reduced morbidity and length of stay in the endoscopic group $[6,7]$. A randomized controlled trial comparing minimally invasive surgery versus endoscopy for necrotising pancreatitis reported reduced incidence of major complications in the endoscopy group (5.9\% Vs $34.4 \%$, risk ratio 0.17 ; $95 \% \mathrm{Cl}, 0.04-0.71$, $P=0.004$ ) [8]. EUS-guided drainage has increasingly been used as first-line treatment in management of PFC.

Double pigtail plastic (DP) stents, fully-covered self-expanding metal stents (FCSEMS) and lumen-apposing covered selfexpanding metal stents (LAMS) have been used in the drainage of PFC. DP stents have equal efficacy in drainage of pseudocysts but they are less efficacious with high adverse event (AE) rates for WON [9]. This often necessitates reintervention and a recent retrospective comparative study (DP vs. FCSEMS vs. LACSEMS) reported that double pigtail stents were the sole negative predictive factor in drainage of WON, on multivariate analysis [9].

FCSEMS are effective in drainage of PFC, especially WON as it allows for debridement but risk of stent migration is between $10 \%$ and $20 \%$. Three retrospective studies reported success rates of $80 \%$ to $94 \%$ for drainage of PFC with a serious AE (SAE) rate of $20 \%$ [10 - 12]

Previous small or retrospective studies have shown that a novel LAMS (Hot AXIOS, Boston Scientific, Boston MA USA) has a higher technical success rate $(98.9 \%)$ than FCSEMS and DP stents with good clinical resolution of collections (92.5\%) [13, 14]. Complication rates are between $5 \%$ and $9.4 \%[9,15,16]$. Here, we report results of a multicenter prospective UK and Ire- land experience with use of LAMS for transluminal drainage of PFC.

\section{Patients and methods}

Retrospective analysis of a prospective, multicenter, observational study of use of LAMS in transluminal drainage of PFC was conducted. All patients who had LAMS placement for PFC from July 2015 to July 2016 were included. Eleven tertiary referral centers for hepatopancreaticobiliary (HPB) medicine participated (St James's University Hospital Leeds, University College London Hospitals, Guy's and St. Thomas' Hospital London, King's College Hospital London, Glasgow Royal Infirmary, Royal Infirmary of Edinburgh, Freeman Hospital Newcastle, Addenbrooke's Hospital Cambridge, Manchester Royal Infirmary, Nottingham University Hospitals NHS Trust, Nottingham, The Adelaide and Meath Hospital, Dublin) in this study. A computed tomography (CT) scan or magnetic resonance imaging (MRI) was repeated at 4 weeks to assess for resolution of the collection and patients were followed up clinically for at least 6 months to assess for recurrence. Data were collected and stored centrally at Leeds Teaching Hospitals NHS Trust. The study protocol was approved by the local R\&D department and information governance department at Leeds Teaching Hospitals NHS Trust. Because this was a prospective case series, it was exempted from ethical approval.

The following definitions were used during data collection. The definitions and explanations are listed in > Table1.

\section{Electrocautery enhanced LAMS (ECE-LAMS)}

The Hot AXIOS device consists of a through-the-scope LAMS delivery system with an electrocautery wire at the tip ( $>$ Fig. 1 and Fig.2). The stent is dumbbell-shaped, self-expanding, fully-covered, and made ot nitinol. The electrocautery wire allows for passage of the catheter without the need to dilate the tract. This means that the endosonographer can deploy the stent in a controlled manner with no requirement for exchanges or use of a guidewire. For PFC drainage, stents are available in 2 sizes (lumen diameter $\times$ length between the flanges): $10 \mathrm{~mm} \times 10 \mathrm{~mm}$ and $15 \mathrm{~mm} \times 10 \mathrm{~mm}$. There are also smaller sizes available for choledochoduodenostomy and gallbladder drainage procedures. The $10-\mathrm{mm}$ saddle length and the dumbbell shape of the stent is designed to appose and anchor the gut to the PFC wall. 
Table 1 Definitions used during data collection.

\begin{tabular}{|l|l|}
\hline Definition & Explanation \\
\hline Technical success & $\begin{array}{l}\text { Appropriate stent position in to the PFC on an } \\
\text { intention to treat basis. }\end{array}$ \\
\hline Clinical success & $\begin{array}{l}\text { Clinical improvement (ie. resolution of symp- } \\
\text { toms for which the procedure was indicated) } \\
\text { and resolution of collection. }\end{array}$ \\
\hline $\begin{array}{l}\text { Stent misplace- } \\
\text { ment }\end{array}$ & $\begin{array}{l}\text { Incorrect placement of the stent in to the cavity } \\
\text { after puncture into the PFC }\end{array}$ \\
\hline $\begin{array}{l}\text { Stent dislodge- } \\
\text { ment }\end{array}$ & $\begin{array}{l}\text { Dislodgement of stent due to manipulation eg, } \\
\text { DEN }\end{array}$ \\
\hline $\begin{array}{l}\text { Direct endoscopic } \\
\text { necrosectomy } \\
\text { (DEN) }\end{array}$ & $\begin{array}{l}\text { Removal of necrotic tissue and debris from the } \\
\text { cyst cavity under direct endoscopic vision. }\end{array}$ \\
\hline Stent migration & $\begin{array}{l}\text { Migration of stent without manipulation. These } \\
\text { were divided into clinically significant or insig- } \\
\text { nificant. }\end{array}$ \\
\hline
\end{tabular}

PFC, pancreatic fluid collection; DEN, direct endoscopic necrosectomy

\section{Drainage Protocol}

Prior to stent insertion, patients were clinically assessed and underwent cross-sectional imaging (CT or MRI) within 2 weeks of the drainage procedure. All patients had walled of necrosis (WON) or pancreatic pseudocysts (PP) as defined by the revised Atlanta Criteria [1]. Antibiotics were continued in patients with infected necrosis, and a single dose of prophylactic intravenous (IV) antibiotic was given during the procedure to patients without infected necrosis, according to local department protocols. Oral antibiotics were continued in some patients according to the local department protocols, for 3 days after the procedure. All procedures were carried out by experienced endosonographers who had experience in EUS-guided cyst-gastrosomy using standard techniques.

At EUS, the collection was assessed using a therapeutic linear echoendoscope and ultrasound workstation (Olympus UCT240/260, Olympus EU ME-2, EG-3870UTK/3270UK, Pentax- Hitachi, Aloka). The size and type of the collection and the percentage of necrosis in the case of WON were assessed. The collection was punctured using the electrocautery wire at the tip of the catheter. Once the catheter was inside the fluid collection, the distal flange of the stent was deployed under EUS guidance. Following this, the catheter was slowly pulled back under EUS guidance until the distal flange tightly apposed the wall of the cyst cavity. Then, the proximal flange was deployed on the luminal side, either under endoscopic view or EUS guidance at the discretion of the endosonographer ( $\vee$ Video 1$)$.

Two sizes of stent $-10 \mathrm{~mm} \times 10 \mathrm{~mm}$ and $15 \mathrm{~mm} \times 10 \mathrm{~mm}-$ were used in this study. Stent size was chosen by the endosonographer at the time of the procedure. No lavage and debridement was performed at the index procedure.

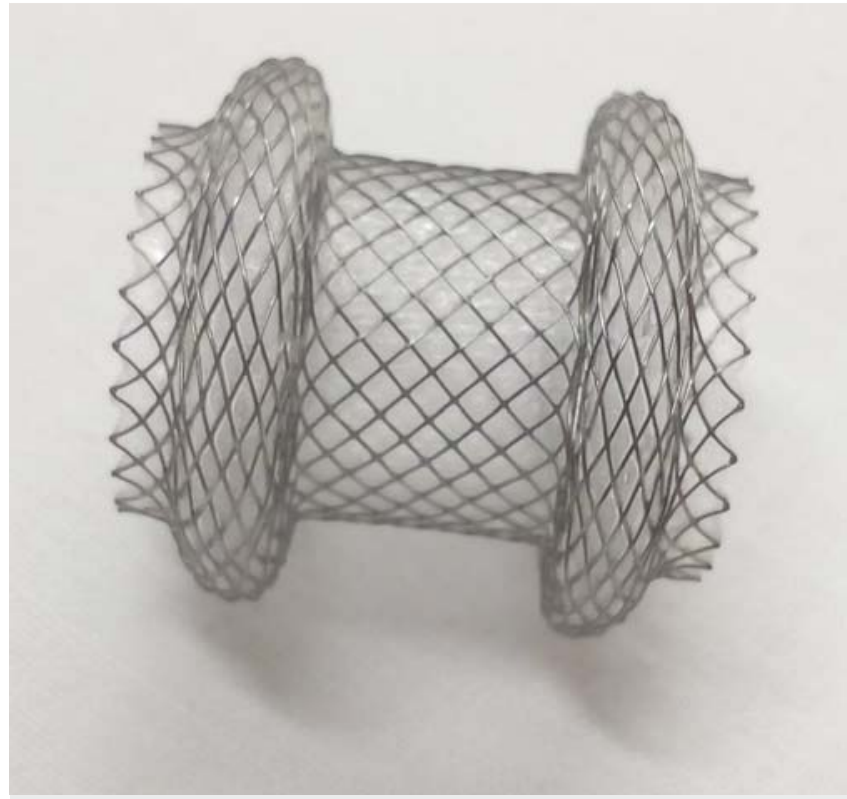

> Fig. 1 Lumen apposing metal stent (LAMS).

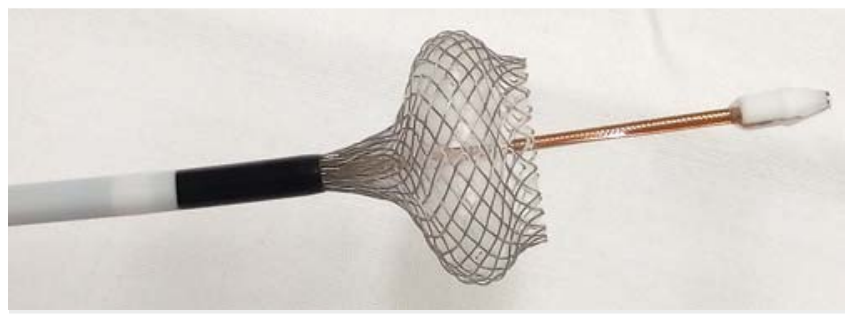

Fig. 2 Partially deployed LAMS.

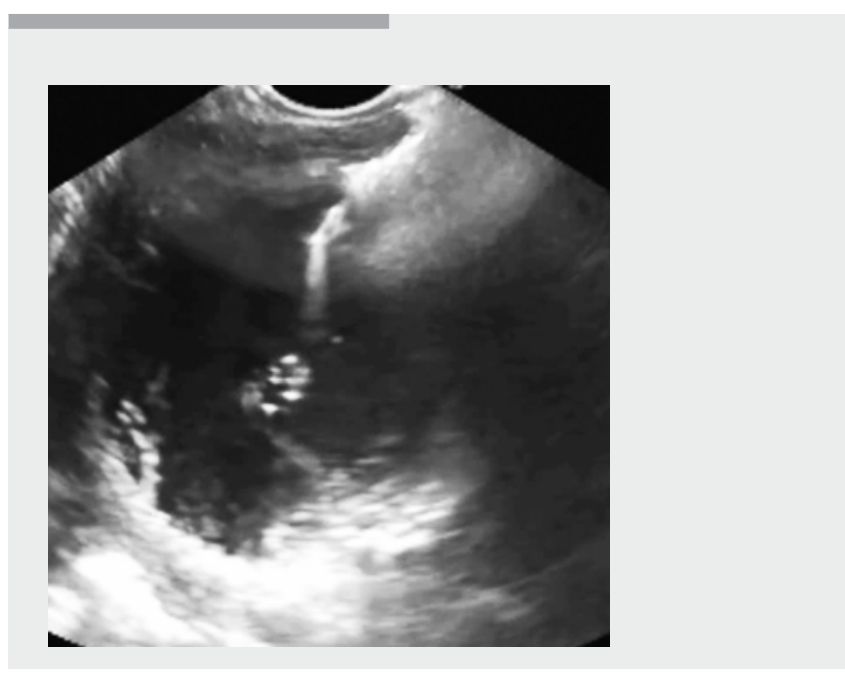

Video 1 Cyst gastrostomy with LAMS. 


\section{Direct endoscopic necrosectomy (DEN) protocol}

Necrosectomy was carried out at the discretion of the attending physician if patients developed evidence of either infected necrosis or stent blockage. The LAMS was usually dilated with a radial expanding balloon (eg: Hercules-cook medical or CRE balloon-Boston Scientific) if a $10 \mathrm{~mm} \times 10 \mathrm{~mm}$ stent was used. The tract was not dilated if a $15 \mathrm{~mm} \times 10 \mathrm{~mm}$ stent was used as the $15-\mathrm{mm}$ diameter allows a gastroscope to be advanced to the collection cavity without dilatation. Necrotic tissue was removed using a basket, Roth Net, ERCP extraction balloon or polypectomy snare. Irrigation was carried out using physiologic saline solution. The procedure was repeated in cases of recurrent sepsis or stent blockage with necrotic material. After DEN, a nasocystic drain was inserted to assist irrigation and drainage of collection at the discretion of the endoscopist.

\section{Outcomes}

The primary outcome of this prospective observational study was assessment of technical and clinical success and AEs associated with insertion of the LAMS for PFC. Secondary outcomes were assessment of collection resolution, number of procedures (necrosectomies, stent changes), stent migration, recurrence at 6 months, and 30- and 90-day mortality.

\section{Results}

\section{Patient characteristics}

One hundred and sixteen patients (78 males, 38 females) from 11 tertiary referral centers from the UK and Ireland were included in the study ( $\vee$ Table 2 ). Median age was 52.5 years (range 16 - 80 years). All patients had confirmed PFC on cross-sectional imaging and the procedures were carried out between July 2015 and July 2016.

Median time from attack of acute pancreatitis to LAMS insertion was 10.7 weeks (range $4-180$ weeks). The etiology of pancreatitis was gallstones $(n=54)$, alcohol $(n=42)$ idiopathic $(n=$ 15), post-endoscopic retrograde cholangiopancreatography $(E R C P)(n=2)$, post-pancreatic surgery $(n=1)$, drug-induced ( $n$ $=1)$, and hyperlipidemia $(n=1)$. Among the 116 patients, indications for drainage were pain in 48 (41\%), sepsis in 49 (42\%), gastric outlet obstruction or early satiety in $28(24 \%)$, obstructive jaundice in $2(2 \%)$ and inadequate previous percutaneous drainage in $2(2 \%)$. The collection resolved partially with percutaneous drainage and patients developed sepsis. Hence, they were considered for EUS-guided cyst gastrostomy. Some patients had more than one symptom.

\section{Procedure}

Seventy (60\%) patients had WON and $46(40 \%)$ had pancreatic pseudocysts. Median maximum collection size on cross-sectional imaging was $11 \mathrm{~cm}$ (range $5-21 \mathrm{~cm}$ ). In patients with WON on EUS assessment, median necrosis within the pancreatic collection was $30 \%$ (range $5 \%-90 \%$ ). Patient and procedure characteristics are displayed in $\mathbf{P}$ Table $\mathbf{2}$.

One hundred and one patients ( $87 \%$ ) received IV antibiotics during the procedure (One center did not prescribe antibio- tics). Of the 116 patients, 76 (66\%) had the procedure under sedation and 40 patients ( $34 \%$ ) had general anaesthesia. All patients except one had free-hand puncture into the cyst cavity without use of an EUS needle and wire. All but one procedure was done without fluoroscopy guidance but it was readily available within the department. The most common site of stent insertion was trans-gastric (112) followed by trans-esophageal (2) and trans-duodenal placement (2).

Seventy-eight patients $(67 \%)$ had $15 \mathrm{~mm} \times 10 \mathrm{~mm}$ stents and $38(33 \%)$ had $10 \mathrm{~mm} \times 10 \mathrm{~mm}$ stents. The stent was dilated in $16(14 \%)$ at the index procedure. The procedure was technically successful in all but one patient (99.1\%). In one patient, the stent was misplaced (distal end of the stent was not placed in to the cyst cavity) and removed and a second stent was placed successfully within the collection during the same procedure.

The procedure was clinically successful in 109 (94\%) patients. Three patients required additional percutaneous drainage because of inadequate drainage at a different site. Median length of stay was 3 days in all patients (Range $0-208$ days). Seventy-seven $(66 \%)$ patients had the procedure while they were inpatients and $39(34 \%)$ had the procedure as a day case procedure. Fifteen procedures (13\%) were done after the patient was admitted to an intensive care unit.

The collection resolved in 109 (94\%) patients and $3(2.5 \%)$ required additional percutaneous drainage. The collection completely resolved in 1 of the 2 patients who died within 30 days. Collection resolution data were not available in 3 patients who were lost to follow up before first follow-up imaging and repeated attempts to contact them have been unsuccessful. Median time to resolution of the collection was 49 days (5$206)$ days. The stent was removed in $99(85 \%)$ patients. In a further 8 patients $(7 \%)$, the stent migrated spontaneously upon resolution of the collection, which was defined as clinically insignificant. The stent could not be removed in 2 patients who died within 30 days of the procedure. In 1 patient who had a buried stent and developed portal hypertensive gastropathy it was deemed that risks of stent removal outweighed the benefits, hence it was decided not to remove the stent. Overall stents have been removed endoscopically or spontaneously migrated in 106/109 (98\%) patients in whom we have data for resolution of the collection. The stents remain in situ in 6 patients $(5 \%)$ in whom repeated attempts at contact have been unsuccessful. When stents were removed, it was done without complication, using either a snare or stent-grabbing forceps. There was one recurrence of a collection during the follow-up period of 6 months.

\section{Procedure-related adverse events}

Procedure-related AEs were divided into early and late AEs. An early $A E$ was defined as any $A E$ that occurred within 7 days after the procedure whereas those occurring more than 7 days after the procedure were defined as late AE. The early SAEs were 5 episodes of sepsis requiring IV antibiotics, 2 requiring emergency DEN, 1 stent blockage with food, 1 stent migration, 1 stent dislodgement during a DEN procedure, and one major hemorrhage requiring 2 units of blood transfusion and gastric artery embolization. These patients developed sepsis despite an- 
Table 2 Patient and procedure characteristics.

\begin{tabular}{|c|c|}
\hline \multicolumn{2}{|l|}{ Patient demographics } \\
\hline Patient characteristics & Number \\
\hline Age (Median, Range) & $52.5(16-80)$ \\
\hline Sex (Male : Female) & $78: 38$ \\
\hline Aetiology & $\begin{array}{l}\text { Gallstones } 54(46.5 \%) \\
\text { Alcohol } 42(36 \%) \\
\text { Idiopathic } 15(13 \%) \\
\text { Post-pancreatic surgery } 1(0.8 \%) \\
\text { Post-ERCP } 2(1.7 \%) \\
\text { Drug-related } 1(0.8 \%) \\
\text { Hyperlipidemia } 1(0.8 \%)\end{array}$ \\
\hline Indication for intervention ${ }^{1}$ & $\begin{array}{l}\text { Pain } 48(41 \%) \\
\text { Infected collection } 49(42 \%) \\
\text { Gastric outlet obstruction/satiety } \\
28(24 \%) \\
\text { Inadequate previous drainage } 2(1.7 \%) \\
\text { Obstructive jaundice } 2(1.7 \%)\end{array}$ \\
\hline \multicolumn{2}{|l|}{ Collection characteristics } \\
\hline Type of collection & $\begin{array}{l}\text { WOPN } 70 \text { (60\%) } \\
\text { Pancreatic pseudocyst } 46 \text { (40\%) }\end{array}$ \\
\hline $\begin{array}{l}\text { Size of collection (median, } \\
\text { range) }\end{array}$ & $11 \mathrm{~cm}(5-21 \mathrm{~cm})$ \\
\hline $\begin{array}{l}\text { Necrosis in WON (median, } \\
\text { range) }\end{array}$ & $30 \%(10-90 \%)$ \\
\hline \multicolumn{2}{|l|}{ Procedure characteristics } \\
\hline Sedation : GA & $76: 40$ \\
\hline Antibiotics & $101(87 \%)$ \\
\hline $\begin{array}{l}\text { Freehand: Wire-guided } \\
\text { puncture into cyst }\end{array}$ & $115: 1$ \\
\hline Site of stent insertion & $\begin{array}{l}\text { Trans-gastric } 111 \text { (96\%) } \\
\text { Trans-duodenal } 3(2.5 \%) \\
\text { Trans-esophageal } 2(1.7 \%)\end{array}$ \\
\hline Stent size & $\begin{array}{l}15 \times 10 \mathrm{~mm} 78(67.3 \%) \\
10 \times 10 \mathrm{~mm} 38(32.7 \%)\end{array}$ \\
\hline Inpatient vs day case & $\begin{array}{l}\text { Inpatients } 77(66 \%) \\
\text { Day cases } 39(34 \%)\end{array}$ \\
\hline $\begin{array}{l}\text { Intraprocedural dilatation } \\
\text { post-stent insertion }\end{array}$ & $16(13.7 \%)$ \\
\hline Technical success & $99 \%$ \\
\hline Clinical success & $94 \%$ \\
\hline \multicolumn{2}{|c|}{$\begin{array}{l}\text { ERCP, endoscopic retrograde cholangiopancreatography; WON, walled off } \\
\text { necrosis } \\
{ }^{1} \text { Some had more than one symptom. }\end{array}$} \\
\hline
\end{tabular}

tibiotics, which resolved with a 3- to 5-day course of IV antibiotics. There was no difference in incidence of sepsis between patients who received antibiotics and those who did not. The patient whose stent migrated required a laparotomy for stent-induced small bowel obstruction. There was one death in which the patient developed pulseless electrical activity (PEA) cardiac
Table 3 Complications.

\section{Serious adverse events}

\begin{tabular}{|l|l|}
\hline Early<7 days & Late $\geq \mathbf{7}$ days \\
\hline $\begin{array}{l}\text { Sepsis requiring IV antibiotics } \\
5(4.3 \%)\end{array}$ & Esophageal fistula $1(0.86 \%)$ \\
\hline $\begin{array}{l}\text { Sepsis requiring emergency DEN } 2 \\
(1.7 \%)\end{array}$ & Buried stent $1(0.86 \%)$ \\
\hline Major hemorrhage $1(0.86 \%)$ & Death $1(0.86 \%)$ \\
\hline Stent migration $1(0.8 \%)$ & \\
\hline Stent blockage with food $1(0.86 \%)$ & \\
\hline Death 1 (0.86\%) & \\
\hline DEN, direct endoscopic necrosectomy & \\
\hline
\end{tabular}

arrest 24 hours after the procedure and died. The cause of death was secondary to pulmonary embolism.

The late SAEs were 1 buried stent and 1 fistula between the esophagus and the subphrenic space. The patient whose stent was buried in a gastric fold developed portal hypertension secondary to splenic vein thrombosis. He developed gastric varices and portal hypertensive gastropathy and it was considered that the risk of serious bleeding outweighed the benefits of stent removal. There was 1 death at 23 days' post-procedure where the patient developed new renal collections away from the existing collection and died of multiorgan failure. The collection into which the stent was inserted had resolved at the time of death, and it was deemed that this mortality was not related to stent insertion. The 30-day, 3-month and 6-month all-cause mortality rates were $1.7 \%, 2.5 \%$, and $3.4 \%$, respectively. Details of the complications are summarized in > Table 3.

Early AEs were predominantly sepsis-related and resolved with IV antibiotics for 3 to 5 days. Two patients required DEN procedures. The late AEs were 1 buried stent and 1 esophagosubphrenic fistula. The patient who developed the fistula was managed with IV antibiotics and required nasojejunal feeding for 30 days. The fistula resolved with the feeding.

\section{WON}

Seventy patients had the procedure for WON. Median collection size was $11 \mathrm{~cm}$ (range $5-20 \mathrm{~cm}$ ) and estimated necrotic volume was $30 \%$ (5\%-90\%). The technical success rate was $100 \%$ and the clinical success rate was $94 \%$ (66 patients). Thirty-seven patients $(37 / 70,53 \%)$ had a median of 2 DEN procedures (range 1-5). The collection resolved in 66 patients (94\%) and median time to collection resolution was 45 days (range, 8-206). The stent was removed in 59 patients (84.28\%). Median length of stay was 8.5 days (range $0-208$ days). Fifteen patients ( $21 \%$ ) had the procedure as a day case procedure. There was no recurrence of WON during the follow-up period of 6 months.

In the WON group, the LAMS migrated spontaneously in 6 patients upon resolution of the collection and none of these were clinically significant. The cumulative SAE rate was $14.2 \%$ (10/ $70)$. Details of the SAE outcomes are described in $>$ Table 4. 
- Table 4 SAE according to type of collection.

\begin{tabular}{|l|l|l|}
\hline Type of SAE & Pseudocyst & $\begin{array}{l}\text { Walled of } \\
\text { necrosis }\end{array}$ \\
\hline Sepsis requiring antibiotics & 1 & 4 \\
\hline Sepsis requiring DEN & 0 & 2 \\
\hline Major hemorrhage & 1 & 0 \\
\hline Stent blockage with food & 1 & 0 \\
\hline Stent migration causing bowel & 0 & 1 \\
\hline obstruction & 0 & 2 \\
\hline Mortality within 30 days & 1 & 0 \\
\hline Buried stent & 0 & 1 \\
\hline Fistula & $4 / 46(8.69 \%)$ & $10 / 70(14.2 \%)$ \\
\hline Cumulative SAE & & \\
\hline SAE, serious adverse event; DEN, direct endoscopic necrosectomy \\
\hline
\end{tabular}

\section{Pseudocysts}

Forty-six patients had the procedure for pseudocysts. Median collection size was $10 \mathrm{~cm}(5-21 \mathrm{~cm})$. The procedure was technically successful in 45 patients (98\%) and was clinically successful in 44 patients. The collection resolved in 42/44 patients (95\%). Median time to collection resolution was 48 days (7203 days) and the stents were removed in 40 patients. Median length of stay was 1 day (Range 0-91 days). Twenty-four (54.5\%) patients had it as a day case procedure. There was one recurrence of pseudocyst during the follow-up period.

There were spontaneous stent migrations in 2 patients upon resolution of collection and these were not clinically significant. The cumulative SAE rate was $8.7 \%$ (4/46; stent blockage with food, buried stent, major hemorrhage and sepsis)

\section{Discussion}

This multicenter study on use of LAMS in PFC confirms that insertion of LAMS is relatively safe and effective for drainage of PFC. The through-the-scope delivery system appears to aid stent deployment into the collection and that was again confirmed in our study, with an overall technical success rate of $99 \%$. Three other retrospective studies have reported similar technical success rates of $97 \%$ to $98.5 \%$ for LAMS [14 - 16]

We observed that the overall median length of stay (LOS) was 3 days ( $0-105$ days); the LOS was significantly lower for pseudocysts with a median LOS of 1 day (Range 0-91 days) compared to 8.5 days in patients with WON (Range 0-105 days, $P=0.01)$. One-third of all patients $(39,34 \%)$ were able to be discharged either on the same day or the day after the procedure. This study demonstrates that endoscopic drainage with LAMS can be safely done as a day case procedure, particularly in the case of a pancreatic pseudocyst. Bang et al, in a retrospective case-control study, reported similar median LOS of 2 days for LAMS drainage [17]. A randomized controlled trial comparing endoscopic drainage (plastic stents) vs. surgical drainage for pseudocysts reported a median LOS of 2 vs. 6 days ( $P<$ $0.001)$ [6]. The TENSION trial which compared surgical vs. endoscopic step-up approach, recently published in abstract form, reported reduced LOS, reduced rate of pancreatic fistula, and significant reduction in costs with the endoscopic step-up approach even though superiority of the endoscopic step-up group was not shown $[7,18]$ LAMS may be associated with reduced morbidity and may be cost-effective in management of PFC compared to surgical drainage. However, randomized controlled trials are needed to establish this.

The technical success rates for WON and pseudocysts were $100 \%$ and $98 \%$, respectively. Clinical success rates were $94 \%$ and $98 \%$. The wide diameter of this LAMS allows for drainage of collections and easy access for DEN. That has been confirmed in our study where 37 patients underwent 74 DEN procedures (median 2, range $1-5$ ). Of the 74 DEN procedures, there was 1 stent dislodgement (1.3\%) during the DEN procedure. In our cohort, $53 \%$ of patients with WON underwent DEN and 1 patient in the pseudocyst group required a further endoscopic procedure for stent blockage. Overall, 78 patients (67\%) with WON had larger-diameter stents $(15 \mathrm{~mm} \times 10 \mathrm{~mm})$ inserted for the drainage procedure. In a recent retrospective study, multivariate analysis demonstrated that clinical success was 6 times more likely if the larger- diameter stent $(15 \mathrm{~mm})$ was used (odds ratio 6.9;1.4-34.5; $P=0.02$ ) [16]. A recent retrospective study of 313 patients comparing plastic stents to FCSEMS to LAMS in drainage of WON reported that the mean number of endoscopic procedures required for collection resolution was significantly lower in the LAMS group compared to the FCSEMS and plastic stent groups ( 2.2 vs. 3 vs. 3.6 , respectively; $P=0.04$ ) [9]. Two retrospective observational studies comparing DP stents, FCSEMS and LAMS reported less efficacy and higher rates of AEs with DP stents compared to FCSEMS and LAMS [9, 12]. Multivariate analysis in 1 retrospective study reported that DP stents were a negative predictive factor in resolution of WON (OR 0.18; $95 \% \mathrm{Cl} 0.06-0.53 ; P=0.002$ ) [9]. This is probably because the solid necrotic matter occludes the narrow diameter of plastic stents and does not allow for DEN procedures through the stents. Necrosectomy in this setting requires additional procedures.

The overall rate of resolution of collections was $94 \%$ and there was only 1 recurrence of a collection during the followup period of 6 months. Two retrospective studies of similar sample size reported a clinical resolution rate of $86 \%$ to $92 \%$ $[14,16]$.

There were 8 (6.4\%) spontaneous migrations of LAMS into the gastric cavity upon resolution of the collection. We found no migrations of the stent into the cyst cavity. That may be due to the negative pressure in the abdomen and compression of intra-abdominal viscera on the stent. A retrospective study of 82 patients reported 2 spontaneous migrations on resolution of the collection [11]. We observed an overall stent migration rate of $7.2 \%$, which appears to be lower than the reported incidence of stent migration with FCSEMS (10\%-20\%) [10-12].

The number of early SAEs reported in this study was 11 $(9.4 \%)$ and late events excluding death were 2 (1.7\%) ( $\triangleright$ Table 3). Thirty- and 90-day mortality rates were low at $1.7 \%$ and 
$2.5 \%$, respectively. Cumulative SAE rates for WON and pseudocyst were $14.2 \%$ and $8.69 \%$, respectively. A recent interim analysis of a randomized controlled trial comparing LAMS and plastic stents in management of WON reported that the risk of AEs was as high as $50 \%$ in the LAMS groups. This included delayed bleeding after insertion, buried stents and obstructive jaundice [19]. However, there was complete resolution of collection in all these patients. Rinninella and Siddqui et al reported similar SAE rates in their retrospective studies $[14,15]$.

The main limitations of this study are lack of a randomized comparator group and differences in follow-up of our patients. There was no set protocol for DEN procedures and these were carried out at the discretion of the endoscopist. It is not clear if collections resolved quicker if patients had a planned DEN procedure within 7 days of the index procedure. Bang et al postulated that this may prevent the late complications reported in their interim analysis [19].

\section{Conclusion}

In summary, we found LAMS to be effective and relatively safe for endoscopic drainage of PFC and WON. Use of LAMS was associated with low morbidity and the observed rates of migration/dislodgement were lower than described in previous studies using FCSEMS. The large diameter of LAMS allows for DEN procedures without dilatation. However, randomized controlled trials comparing LAMS with FCSEMS and plastic stents are needed to assess the therapeutic benefit of LAMS.

\section{Competing interests}

\section{None}

\section{References}

[1] Banks PA, Bollen TL, Dervenis C et al. Classification of acute pancreatitis - 2012: revision of the Atlanta classification and definitions by international consensus. Gut 2013; 62: $102-111$

[2] Kahaleh M1, Shami VM, Conaway MR et al. Endoscopic ultrasound drainage of pancreatic pseudocyst: a prospective comparison with conventional endoscopic drainage. Endoscopy 04 2006; 38: 355-359

[3] Binmoeller KF, Seifert H, Walter A et al. Transpapillary and transmural drainage of pancreatic pseudocysts. Gastrointest Endosc 1995; 42: $219-224$

[4] Varadarajulu S, Rana SS, Bhasin DK. Endoscopic therapy for pancreatic duct leaks and disruptions. Gastrointest Endosc Clin N Am 2013; 23 : $863-892$
[5] Singhal S, Rotman SR, Gaidhane M et al. Pancreatic fluid collection drainage by endoscopic ultrasound: an update. Clin Endosc 2013; 46: $506-514$

[6] Varadarajulu S, Bang JY, Sutton BS et al. Equal efficacy of endoscopic and surgical cystagastrostomy for pancreatic pseudocyst drainage in a randomized trial. Gastroenterology 2013; 145: 583-590

[7] van Brunschot S, van Grinsven J, van Santvoort HC et al. Endoscopic or surgical step-up approach for infected necrotising pancreatitis: a multicentre randomised trial. Lancet 2017: doi:10.1016/S0140-6736 (17)32404-2

[8] Bang JY, Arnoletti JP, Holt BA et al. Minimally invasive surgery vs endoscopy randomised (MISER) trial for necrotising pancreatitis. United Eur Gastroenterol J 2017; 5: 5 S

[9] Siddiqui AA, Kowalski TE, Loren DE et al. Fully Covered Self-Expanding Metal Stents Versus Lumen Apposing Fully Covered Self-Expanding Metal Stent Versus Plastic Stents for Endoscopic Drainage of Pancreatic Walled-off Necrosis: Clinical Outcomes and Success. Gastrointest Endosc 2017; 85: $758-765$

[10] Huggett MT, Oppong KW, Pereira SP et al. Endoscopic drainage of walled-off pancreatic necrosis using a novel self-expanding metal stent. Endoscopy 2015; 47: 929-932

[11] Chandran S, Efthymiou M, Kaffes A et al. Management of pancreatic collections with a novel endoscopically placed fully covered self-expandable metal stent: a national experience (with videos). Gastrointest Endoscopy 2014; $2: 1$ - 9

[12] Varquez-sequeiros E, Baron TH, Perez-Miranda M et al. Evaluation of the short- and long-term effectiveness and safety of fully covered self-expandable metal stents for drainage of pancreatic fluid collections: results of a Spanish nationwide registry: Gastrointest Endosc. 2016 Sep84: $450-457$

[13] Shah RJ, Shah JN, Waxman I. Safety and efficacy of EUS guided drainage of PFC with lumen apposing covered self -expanding metal stents. Clin Gastroenterol Hepatol 2015; 13: 747 -752

[14] Rinninella E, Kunda E, Dollhopf E et al. EUS-guided drainage of pancreatic fluid collections using a novel lumen-apposing metal stent on an electrocautery-enhanced delivery system: a large retrospective study. Gastrointest Endosc 2015; 82: 1039 - 1046

[15] Siddiqui A, Adler DG, Nieto J et al. EUS-guided drainage of peripancreatic fluid collections and necrosis by using a novel lumen-apposing stent: a large retrospective, multicenter U.S. experience. Gastrointest Endosc 2016; 83: 699-707

[16] Sharaiha RZ, Tyberg A, Khashab MA et al. Endoscopic therapy with lumen-apposing metal stents is safe and effective for patients with pancreatic walled-off necrosis. Clin Gastroenterol Hepatol 2016; 14 : $1797-1803$

[17] Bang JY, Hasan MK, Navaneethan U et al. Lumen-apposing metal stents for drainage of pancreatic fluid collections: When and for whom? Dig Endosc 2017; 29: 83-90

[18] van Brunschot S, van Stanvoort HC, Gooszen HG et al. Endoscopic or surgical step-up approach for necrotizing pancreatitis, a multi-center randomized controlled trial. OP004 UEGW abstract 2016

[19] Bang JY, Hasan M, Navaneethan U et al. Lumen-apposing metal stents (LAMS) for pancreatic fluid collection (PFC) drainage: may not be business as usual. Gut 2016; 66: $2054-2056$ 